\section{FORMAÇÃO EM EDUCAÇÃO FÍSICA: EXPERIÊNCIAS DE INTEGRAÇÃO ENSINO-SERVIÇO NA ATENÇÃO BÁSICA EM SAÚDE}

\author{
TRAINING IN PHYSICAL EDUCATION: EXPERIENCES OF SERVICE/LEARNING \\ INTEGRATION IN PRIMARY HEALTH CARE
}

FORMACIÓN EN EDUCACIÓN FÍSICA: EXPERIENCIAS DE INTEGRACIÓN
DOCENCIA-SERVICIO EN LA ATENCIÓN PRIMARIA DE SALUD

Alessandro Rovigatti do Prado*, Yara Maria de Carvalho*

\section{Palavras-chave}

Capacitação de recursos humanos em saúde.

Educação em saúde. Sistema Único de Saúde (SUS).
Resumo: Este artigo pretende problematizar sobre a formação em saúde, partindo de experiências de um grupo de estudantes de Educação Física no PET-Saúde USPCapital no período de 2010 a 2012. Entendemos que a formação dos profissionais de saúde precisa estar mais comprometida com o SUS, mais próxima da prática profissional e do contexto social das pessoas e dos coletivos, o que é possível numa aproximação com a atenção básica em saúde. Destarte, acreditamos que o PET-Saúde é uma potente estratégia no processo formativo em saúde, já que o Programa apresenta cenários de integração ensino-serviço com valiosas vivências.

\section{Keywords}

Training of human resources in health. Health Education. Unified Health System.

\section{Palabras clave} Capacitación de recursos humanos en salud.

Educación en salud. Sistema Único de Salud (SUS).

\begin{abstract}
This essay aims to discuss training in health, starting from experiences of a group of Physical Education students at program PET-Saúde USP-Capital in 2010-2012. We understand that the training of health professionals has to be more committed to Brazil's Unified Health System and closer to professional practice and the social context of individuals and groups, which is possible under an approach focused on primary health care. Thus, we believe that PET-Saúde is a powerful strategy in health training, since the program provides service-learning integration scenarios with valuable experiences.
\end{abstract}




\section{CONSIDERAÇÕES PRELIMINARES}

Por que discutir formação em saúde? Dessa questão partimos para outra: o que aprendemos sobre o SUS (Sistema Único de Saúde) durante a graduação em Educação Física? Aliás, por que aprender sobre o SUS durante a graduação?

Esses são os questionamentos norteadores do presente artigo, os quais visamos explorar a partir das experiências de integração ensino-serviço de estudantes de Educação Física, durante atividades desenvolvidas no Programa de Educação pelo Trabalho (PETSaúde) de 2010 a 2012, no projeto da Universidade de São Paulo (USP), USP-Capital, em unidades básicas de saúde (UBS) localizadas no distrito Butantã.

O PET-Saúde decorre de estratégia política que visa fortalecer a integração ensinoserviço na atenção básica em saúde, priorizando atividades em grupos multiprofissionais dentro do serviço público de saúde. Trata-se de uma iniciativa do Ministério da Saúde que incentivou parcerias entre as Secretarias Municipais de Saúde e as Instituições de Ensino Superior (IES), contribuindo para aproximações entre a universidade, o serviço público de saúde e os usuários do SUS.

Pretendemos chamar a atenção para a questão da experiência no processo formativo em saúde, principalmente em Educação Física, que necessita estar mais integrada ao SUS, em consonância com o cenário político, em cooperação com outras categorias profissionais, e em articulação com a realidade social e com as necessidades de saúde e de cuidado da população, tendo em vista profissionais qualificados. Sendo assim, a ideia nuclear deste ensaio é discutir, a partir de relatos de experiências no Programa, a importância do SUS para a formação em saúde, sobretudo para a área da Educação Física. Por esse motivo, o PETSaúde pode ser considerado um importante ingrediente para a formação, haja vista a instigante e rica vivência de integração ensino-serviço na atenção básica em saúde.

\section{ALGUMAS CONTEXTUALIZAÇÕES REFERENTES AO PET-SAÚDE}

\subsection{O PET-Saúde como fruto de mobilizações e estratégias políticas}

Para mencionar de forma breve o cenário em ensino superior no que diz respeito às mobilizações da área da Educação Física para as demandas de formação em articulação com o SUS, cabe destacar as palavras de um grupo de pesquisadores que se dedica a pesquisas com o tema "Políticas de formação em Educação Física e saúde coletiva": "[...] as fortes tradições técnico-esportiva e médico-científica têm gerado resistências consideráveis ao processo de reformulação de currículos e de práticas docentes em larga escala em formação superior nessa área [em Educação Física]" (FRAGA et al., 2012, p. 378). Há um tradicionalismo impregnado na área que persiste em diversas categorias - epistemológica, política e científica - e que transmite um certo modo de saber e de fazer que reproduz, muitas vezes de forma acrítica, determinados procedimentos incoerentes com a demanda social de saúde.

Em geral, no campo da saúde, o ensino superior tradicionalmente tem sido orientado, de modo predominante, pelo modelo biomédico, pautado na doença e na reabilitação, com uma abordagem centrada em conteúdos e na pedagogia da transmissão, com carga horária 
que prevalece a oferta de disciplinas teóricas na perspectiva técnico-científica. A dimensão prática, na qual o estudante tem contato com as pessoas que necessitam de cuidados ou de atendimentos, tem prevalecido da metade para o final dos cursos. Esse modelo de formação, que, de certo modo, segue uma racionalidade centrada no hospital, não tem sido suficiente para a demanda que o tema "saúde" envolve, sobretudo quando se trata de atenção básica (CARVALHO; CECCIM, 2009, FEUERWERKER, 2002).

Na recente história do SUS, a integração ensino-serviço, que tem respaldo no processo histórico da reforma sanitária no Brasil, tem sido satisfatoriamente concebida como um modo interessante de pensar a formação e a educação em saúde (CARVALHO; CECCIM, 2009, FONSÊCA; JUNQUEIRA, 2014). Um marco importante foi a determinação em nossa Carta Constitucional de que a saúde é dever do Estado e direito dos cidadãos (BRASIL, 1988), o que colocou o SUS como referência no processo de formação do profissional da saúde com vistas a garantir a articulação entre o sistema, as necessidades de saúde da população e a formação do profissional.

Como mostram Carvalho e Ceccim (2009) e Fonsêca e Junqueira (2014), com a regulamentação do SUS nos anos 1990, os debates e mobilizações em torno de mudanças curriculares que ocorreram nessa década para as profissões da saúde visaram adequar o perfil do trabalhador à realidade do país, que, por sua vez, configurava-se com as novas referências políticas. Para tanto, cabe mencionar a criação das Diretrizes Curriculares Nacionais (DCN), em 1997, em contraponto ao currículo mínimo, tendo em vista a flexibilização na organização curricular que, em geral, colocou o desafio de preparar profissionais para lidar com as realidades socioculturais do país, requisitando pessoas críticas, com senso de responsabilidade social e de liderança.

Todavia, lançadas as referências políticas, as IES não se adequaram às novas orientações - por motivos diversos, como de ordem estrutural, política e financeira -, sendo necessárias outras ações; havia falta de estímulo às novas iniciativas, como foi apontado, no ano 2000, na 11a Conferência Nacional de Saúde. Em outras palavras, a formação ainda estava distante da realidade do SUS, cabendo adequação de currículos por parte das IES, de acordo com as DCN. Nas palavras de Fonsêca e Junqueira (2014, p. 35): "[...] as DCN apontam para uma nova fase da relação entre o ensino e os serviços de saúde".

No entanto, é preciso destacar também outras mobilizações importantes que contribuíram para o desenvolvimento e para o fortalecimento de ações acerca do processo de formação, como o PROMED (Programa de Incentivo às Mudanças Curriculares nos Cursos de Medicina), iniciado em 2002, de elaboração conjunta dos ministérios da Saúde e da Educação, que incentivou escolas médicas para mudanças curriculares com referência no SUS; e o Pró-Saúde (Programa Nacional de Reorientação da Formação Profissional em Saúde), implementado em 2005, sendo fruto novamente de trabalho conjunto interministerial, dessa vez com uma aproximação mais sólida, envolvendo diversas secretarias: SESu (Secretaria de Educação Superior), SETEC (Secretaria de Educação Profissional e Tecnológica) e INEP (Instituto Nacional de Estudos e Pesquisa Anísio Teixeira), por parte do Ministério da Educação, e a SGTES (Secretaria de Gestão do Trabalho e da Educação na Saúde), por parte do Ministério da Saúde. Com relação ao Pró-Saúde, destaque para o incentivo em ampliar as relações entre a universidade e o serviço de saúde, dando continuidade, então, a uma fase promissora com relação à formação em saúde (FONSÊCA; JUNQUEIRA, 2014). 
Em suma, essas iniciativas, que cooperaram com políticas de educação e de saúde, visaram, além de qualificar a formação para o SUS, contribuir para a consolidação de um sistema de saúde fortalecido dentro da sociedade. E o PET-Saúde, então, aparece como uma das estratégias políticas dessa parceria interministerial.

\subsection{PET-Saúde: singularidades}

Considerando a atual estrutura do SUS, a atenção básica à saúde está configurada como um campo que lida com um amplo e heterogêneo conjunto de problemas e necessidades de saúde, os quais demandam a produção de intervenções singularizadas e integradas aos diferentes níveis do sistema de saúde (MASSUDA, 2008). Nesse sentido, a Estratégia de Saúde da Família (ESF) se apresenta como um modo de estruturar e consolidar os princípios organizativos do SUS, tendo em vista que compreende um modelo de atenção básica que opera na esfera comunitária com respaldo na unidade familiar (ANDRADE et al., 2009). É nesse cenário de atenção básica que se insere o PET-Saúde.

Nesse sentido, embora ainda restrito a pequena parcela de estudantes e instituições, o PET-Saúde, de um lado, é uma estratégia em potencial para responder às proposições do Ministério da Saúde com relação ao SUS e, de outro, supre a necessidade de aproximar ensinoserviço e enfrentar os impasses da distância entre a universidade e a sociedade. É uma iniciativa recente e que efetivamente tem aberto e ampliado as relações e os diálogos com o SUS, principalmente durante a formação, pois se trata de um período crítico na vida do estudante.

Os objetivos do programa enfatizam formar profissionais de saúde para o SUS; fortalecer a integração ensino, serviço e comunidade; estimular a intersetorialidade e a produção de conhecimento voltada para a atenção básica; e assegurar uma forma de qualificar o serviço de saúde, entre outros (BRASIL, 2008). Embora sejam essenciais para a formação em saúde, as atividades e discussões que o PET-Saúde promove ainda são, em muitos cursos, extracurriculares e por esse motivo nos referimos ao Programa como sendo um potente ingrediente para a formação em saúde.

O Programa está espalhado pelo Brasil em diferentes versões, e o que caracteriza essa diversidade são as peculiaridades políticas locais, as condições físicas (estrutura e equipamentos), recursos financeiros e humanos de cada instituição de ensino, bem como dos serviços de saúde. No PET-Saúde da Universidade de São Paulo (USP-Capital), a Educação Física foi inserida na edição de 2010-2011, compondo um grupo juntamente com outros nove cursos da área da saúde (Enfermagem, Odontologia, Ciências Farmacêuticas, Medicina, Nutrição, Fisioterapia, Terapia Ocupacional, Fonoaudiologia e Psicologia). No período de 2010-2012, a que se refere este trabalho em específico, estiveram vinculados ao projeto seis Unidades Básicas de Saúde (UBS) localizadas no distrito Butantã, zona oeste da cidade de São Paulo, e 92 estudantes bolsistas de todas as áreas da saúde da USP-Capital.

Das atividades gerais, podemos destacar: o reconhecimento do território de cada UBS, momento em que os estudantes, junto com os profissionais de saúde, conhecem a realidade das condições de vida e de saúde da população; discussão e planejamento das atividades de pesquisa e intervenção a serem priorizadas ao longo do projeto; encontros quinzenais, em que cada curso se reunia separadamente para estudo e preparação das atividades específicas, dos núcleos de saberes e práticas a serem desenvolvidas junto com os estudantes das outras áreas; 
e os seminários e encontros reunindo todos os envolvidos com intuito de trocar experiências e aprofundar o debate a respeito do projeto propriamente dito.

\section{A APROXIMAÇÃO DOS ESTUDANTES NO SERVIÇO PÚBLICO DE SAÚDE: AS EXPERIÊNCIAS!}

Descreveremos, na sequência, cinco atividades desenvolvidas no Programa. Ao final deste tópico, uma conclusão geral com a seguinte questão: como a experiência de integração ensino-serviço no PET-Saúde pode qualificar a formação em saúde? Nosso intuito é conduzir o leitor ao cenário de práticas e, ao mesmo tempo, mostrar, em linhas gerais, a participação dos estudantes de Educação Física em algumas vivências de integração ensino-serviço que 0 PET-Saúde promoveu no período de 2010-2012.

\subsection{Feira do amor: uma campanha contra a AIDS}

Junto com os profissionais da UBS, houve participação dos estudantes no planejamento e na execução de uma campanha, com prestação de informações para a comunidade sobre a AIDS. As atividades aconteceram em um espaço na comunidade, numa espécie de feira, onde houve pontos para esclarecimento de dúvidas, demonstrações lúdicas da utilização de preservativos e de métodos anticoncepcionais, e filmes educativos e ilustrativos sobre a AIDS.

O evento foi importante para educação e também para prevenção de doenças, na medida em que aproximou a população das atividades da UBS e contribuiu para a prestação de informação sobre uma forma de adoecer que pode ser facilmente evitada. Destaque aqui para o trabalho em equipe multiprofissional.

\subsection{Contra as impurezas}

Por meio de estudo do território, mediado por um questionário aplicado por estudantes e profissionais da saúde em uma comunidade no entorno da UBS, foi identificado que a principal preocupação dos moradores era com o acúmulo de lixo em locais inapropriados e, com isso, a atração de transmissores de doenças contagiosas. Com base nesse levantamento, desenvolvemos um planejamento visando às intervenções.

O serviço de saúde, junto com os estudantes do PET-Saúde, investiu nesse projeto e formulou duas propostas: a primeira esteve ligada ao aspecto informativo e educativo voltado para a comunidade, basicamente centrado na confecção de folders a serem distribuídos pelas casas. A ideia era anunciar o problema para a população, trazendo esclarecimentos sobre o acúmulo de lixo e suas consequências, para que fosse possível conscientizar as pessoas a respeito do despejo pessoal e comunitário do lixo; a segunda tinha como foco a coleta do lixo: fizemos contato com empresas para disponibilizar infraestrutura necessária para que 0 lixo fosse coletado. Embora tenhamos saído frustrados diante dessa intervenção, haja vista os resultados serem de médio e longo prazo, conseguimos abrir outra frente a ser trabalhada com a comunidade, a saber, a reciclagem do lixo.

A preocupação, no entanto, advinha de um reconhecimento multifatorial sobre 0 problema. De nada adiantaria receitar aspirinas ou educar a pessoa a lavar as mãos antes 
das refeições sendo que havia lixo na porta das casas, uma questão social, coletiva, e que envolve ações e políticas. 0 controle social e a mobilização das pessoas para solucionar 0 caso requerem orientações e intermediações que os profissionais da UBS podem fazer. Mas a questão aqui não se fixa nos ratos, ou nos micróbios, e em seus efeitos no corpo biológico, mas, sobretudo, nas questões associadas ao cotidiano, aos modos de viver dessas pessoas.

\subsection{Caminhando ao encontro da saúde}

Em outro exemplo, agentes comunitários, profissionais do Núcleo de Apoio à Saúde da Família (NASF) e estudantes inseridos no Programa participaram de um grupo de caminhada que acontecia em uma praça na comunidade. $O$ grupo, além de promover o encontro de várias pessoas, era uma espécie de difusão de serviços da UBS, pois muita gente que não frequentava a unidade básica aparecia para apreciar e usufruir o grupo e, ao ter contato com os agentes, profissionais e estudantes, recebiam indicações e orientações para consulta, serviços e outras informações ou atividades da unidade.

O grupo de caminhada acontecia em dois locais da comunidade e era gerenciado pelo NASF em parceria com a Estratégia Saúde da Família (ESF). Para os estudantes, foi um momento bem construtivo, pois sempre havia prestação de informações e necessidade de articular conhecimentos de outras áreas.

Como pode ser notado, a ideia era propiciar um momento de escuta da população em que a caminhada foi um pretexto. Não eram prioridade os procedimentos antropométricos para "avaliar" a saúde ou o condicionamento físico, mas a continuidade de um momento em que as pessoas pudessem se encontrar e se sentir parte de um grupo. Essa é uma questão que dificilmente pode ser proposta em sala de aula, pois remete a um aprendizado relativo a sensibilidade e a um vínculo que só acontece e persiste ali onde o grupo existe.

\subsection{Conectando pessoas}

A partir de um projeto que visou melhorar a acessibilidade e a comunicação entre o serviço de saúde e a comunidade, foi realizado um estudo do território. Nesse percurso, juntamente com o aporte de dados de outras pesquisas vinculadas à UBS e entrevistas feitas com a comunidade, foi possível identificar algumas necessidades de saúde e dificuldades enfrentadas pela população local para, então, pensar e planejar uma intervenção.

No que se refere à comunidade especialmente, cabe ressaltar que, além de estar distante da unidade de saúde, estava isolada geograficamente, seu único acesso à UBS era através deuma rodovia federal, muito movimentada, que não oferecia condições seguras para o tráfego de pedestres.

Visando facilitar o acesso, os estudantes do PET inseridos neste território fizeram reuniões com a equipe de saúde responsável pela área a fim de definirem e planejarem três principais ações: criação de um canal de comunicação Escola-UBS, trazendo o serviço de saúde mais próximo da população e das crianças; criação de oficina de brincadeiras, na qual realizamos um trabalho junto com os agentes comunitários para desenvolver atividades com crianças; e ofício para a extensão da passarela situada junto à rodovia, que visava facilitar o acesso a uma área de lazer, ampliando as opções de atividades culturais e recreativas. 
Esse obstáculo à comunicação e ao acesso entre os equipamentos sociais também pode acentuar uma exposição a outros riscos para a saúde, envolvendo mais pessoas e mais problemas. Além disso, contribui para dificultar ou até mesmo inviabilizar um projeto terapêutico e, ainda, afetar a disposição e o interesse da criança na escola.

\subsection{0 corpo em arte}

Foi criado um grupo de práticas corporais com dança para a comunidade em uma UBS. A ideia era acolher, sem restrições, qualquer pessoa que tivesse interesse em participar. Pensando na questão do acesso ao cuidado com o corpo, a comunidade tinha pouco contato com as práticas corporais, e a ideia do grupo, em princípio, era a de proporcionar um encontro no qual 0 aprendizado iria além do mexer 0 esqueleto e 0 de experimentar coreografias, mas que garantisse uma proximidade maior com a arte, especialmente com a dança.

Nesse sentido, as atividades desenvolvidas, além de trabalhar o condicionamento físico, as habilidades motoras, e as atividades rítmicas e lúdicas, promoviam o encontro de pessoas (agente comunitário, profissionais, estudantes e usuários), no qual a troca de experiência, o trabalho em equipe e a criação de vínculos ensinavam a todos. A atividade colaborou com 0 funcionamento da UBS e levou o grupo a se apresentar em um evento cultural na universidade.

Como essas experiências de integração ensino-serviço no PET-Saúde podem qualificar a formação em saúde?

O que é importante enfatizar a partir dessas participações no Programa, aqui na perspectiva dos estudantes, além de conhecer o serviço público de saúde local, o SUS, e de poder trabalhar junto com a equipe de saúde e com a comunidade, é a oportunidade de estar inserido no ambiente de trabalho, conhecendo a prática profissional no nível de atenção básica em saúde e suas exigências no que se refere às formas de atuação. Fonsêca e Junqueira (2014) também destacam a relevância deste tipo de experiência, sendo importante no processo de formação do futuro profissional da saúde, pois, muitas vezes, permite aos estudantes conhecer a realidade dos fatores que determinam as condições de saúde da população, o que contribui para que eles atribuam significados diferentes para as ações em saúde, especialmente considerando a distância histórica entre as categorias profissionais.

De modo geral, ao analisar essas vivências sob a perspectiva do trabalho de Ceccim e Fewerverker (2004), podemos afirmar que as experiências no PET-Saúde resultam da composição ensino+serviço+comunidade. Para o "ensino", considerando a inserção do estudante no cenário profissional mais a aproximação dos usuários, profissionais, gestores e docentes da universidade, temos um conjunto que contribui para reforçar o aprender e o ensinar mútuo entre as partes, todos ensinam e todos aprendem. 0 "serviço" não toca apenas nas práticas de atenção, mas lida também com a gestão, com as políticas, com a organização e com a interação de saberes. É no serviço que ocorre a intermediação entre o ensino e a esfera da comunidade. E é na "comunidade" que as coisas acontecem; acontecem no sentido político do direito social e do dever do Estado, indo além da interação entre as pessoas e das suas necessidades, mas tocando no controle social, da participação da população no debate e na implementação de estratégias, sempre pautada em uma responsabilidade que contribua para a produção de saúde com relevância social. 
Portanto, as experiências de integração ensino-serviço no PET-Saúde qualificam a formação na medida em que elas complementam as atividades teóricas da graduação, além de explorar e contribuir para desenvolver habilidades de comunicação, liderança, responsabilidade social e espírito crítico.

\section{A INTEGRAÇÃO ENSINO-SERVIÇO A PARTIR DO PET-SAÚDE COMO UM POTENTE INGREDIENTE NO PROCESSO FORMATIVO}

Na literatura científica, constam publicados diversos relatos feitos a partir de experiências no PET-Saúde em todo o país. A ideia neste tópico é, juntamente com alguns destes relatos, problematizar as experiências de integração ensino-serviço que ocorreram a partir do PETSaúde, destacando o Programa como um potente ingrediente no processo formativo, sobretudo em Educação Física.

Os autores Prado e Carvalho (2012), Alonso e Carvalho (2012), Campos et al. (2012), Dias et al. (2012), Ferreira et al. (2012) e Teixeira et al. (2012) enfatizam que a experiência de inserção discente no PET-Saúde propicia um ambiente de aprendizado ímpar ao aproximar estudantes de profissionais e usuários do SUS na esfera da comunidade. Isso contribui para que as atividades sejam diversificadas e com amplas possibilidades de questões a serem discutidas tendo em vista o grupo multiprofissional, a experiência de vida e profissional de cada integrante e, também, as peculiaridades locais de cada serviço de saúde. Em outros termos, cabe destacar a diversificação do cenário de ensino-aprendizagem como estratégia pedagógica para explorar habilidades como comunicação, liderança, resolução de problemas e relações interprofissionais.

Outro ponto que os autores supracitados destacam, enfatizando a integração ensinoserviço, é o diálogo interdisciplinar e interprofissional. Embora exista a dificuldade da interação entre as categorias profissionais, a experiência interprofissional, quando bem operada e feita sob supervisão, coloca o estudante em contato com diferentes olhares sobre as situações e sobre os casos, propiciando uma vivência pedagógica com o princípio da integralidade do SUS. No momento em que esse horizonte do olhar se expande, o repertório para a atuação profissional tende a ser ampliado.

Ao questionarem a estrutura curricular, diversos autores que relataram sua participação no PET-Saúde reconhecem a importância de experimentar a diversificação de cenário de ensinoaprendizagem, principalmente em aproximação com a realidade social, que pode contribuir para ampliar a visão crítica sobre a atuação voltada para o coletivo ou para a comunidade (ALMEIDA et al., 2012, TANAKA et al., 2012, ZEM-MASCARENHAS et al., 2012).

Abrahão et al. (2011), Morais et al. (2013) e Sousa Neto et al. (2012) mostram, em geral, a partir de suas experiências no Programa juntamente com a passagem pela graduação em saúde, que a educação participativa, no sentido de formar profissionais atuantes e conscientes da realidade, ao longo do período de formação, precisa ganhar espaço diante do aprendizado que reduz o conhecimento somente ao técnico-científico, sobretudo quando se trata de conhecimentos essenciais para compor ações direcionadas para pessoas.

Isso soa de forma mais condizente com uma formação diferenciada, atenta à realidade de saúde no nível local e global, preocupada com ações responsáveis em resposta às necessidades de saúde da população, isto é, alinhadas com os princípios do SUS, como 
reforçam Carvalho e Ceccim (2009, p. 155): "a configuração das dimensões técnica, científica, pedagógica, ética, humanística e política deve pertencer ao projeto de profissionalização requerido pelo país e por suas instâncias de participação e controle social".

A vivência para o estudante de Educação Física na atenção primária, portanto, pode despertar outros modos de compreensão do processo saúde-doença, os quais contribuem para uma qualificação diferenciada do profissional, já que complementa a formação no curso de graduação, que, em geral, segue a linha tradicional do pensamento biomédico em saúde (CYRINO et al., 2012, SANTOS et al., 2011, SILVA et al., 2012). Ao aproximar a Educação Física do contexto social, das demandas de saúde, estamos também repensando as práticas de saúde, a formação e a legitimidade enquanto profissão (CARVALHO et al., 2013). E, no que diz respeito ao seu objeto central, o corpo em movimento, também estamos possibilitando outros olhares, olhares esses que extrapolam os limites de um corpo biológico estabelecido cientificamente.

E essa aproximação da Educação Física também nos faz refletir sobre a formação em saúde, pois seu núcleo de saberes lida diretamente com temas complexos e instigantes, como, por exemplo: a medicalização da sociedade; a promoção da saúde; a prescrição de atividade física e exercício físico; a sexualidade; o corpo em movimento; os critérios para formar grupos de atividade física; a diferença conceitual entre atividade física, práticas corporais, lazer e recreação; o imaginário da vida ativa; e a utilização de fármacos.

Com base no exposto e nas experiências relatadas no tópico anterior, é preciso afirmar que há outras dimensões do ser humano que colocam obstáculos no entendimento de suas necessidades de saúde e que instigam a pensar que existem variações subjetivas que, muitas vezes, tolhem as intervenções mais técnicas em relação ao cuidado. Nesse sentido, enquanto profissionais da saúde, é preciso ser inventivos, temos que considerar e propor outros modos de interpretar ou mesmo de pensar a saúde das pessoas, que não é só por meio da ciência, mas também por meio da arte, da filosofia e da sabedoria popular, por exemplo.

\section{ALGUMAS NOTAS FINAIS: EM DEFESA DO SUS PELA FORMAÇÃO EM SAÚDE}

Bondía (2002) propõe pensar a educação de uma forma interessante, através do par experiência/sentido. Para o autor, experiência é "[...] o que nos passa, o que nos acontece e o que nos toca. Não o que se passa, ou o que acontece ou o que toca. A cada dia se passam muitas coisas, porém, ao mesmo tempo, quase nada nos acontece" (BONDÍA, 2002, p. 21). Portanto, o sentido da experiência, que é unicamente atribuído pelo sujeito que a vivenciou, representa aquilo que nos comove e nos provoca a agir de forma intensa em determinada circunstância, tocando cada pessoa de forma diferente. Esse sentido da experiência na esfera do aprendizado, juntamente com nossa curiosidade, deveria ser o motor dos processos educativos. "[...] a experiência não é um caminho até um objetivo previsto, até uma meta que se conhece de antemão, mas é uma abertura para o desconhecido [...]" (BONDÍA, 2002, p. 28).

É essa dimensão da experiência e do aprendizado que desejamos para a formação em saúde, em específico aqui para a Educação Física, pois no serviço (onde se encontra o ambiente de trabalho e de aprendizado) a dinâmica da rotina e do processo de trabalho se altera insistentemente, requerendo sensibilidade e profissionalismo para atuar de modo adequado e responsável. 
Com base no tema da experiência, formulamos a seguinte reflexão: sendo a experiência uma abertura para o desconhecido, por que a passagem pelo PET-Saúde pode se tornar uma "travessia perigosa"? A abertura para o desconhecido ocorre tendo em vista, por exemplo, que a área da saúde, no que diz respeito à formação, sobretudo em Educação Física, ainda não está comprometida com o SUS em grande parte de seus projetos pedagógicos. Porém, essa abertura para o desconhecido, que ocorre muitas vezes no referido programa, precisa ser considerada com bons olhos, já que a diversificação dos cenários de práticas profissionais e de ensino-aprendizagem propicia valiosas vivências na atenção básica.

Sim! Essa abertura para o desconhecido tornou a "travessia perigosa". Se retomarmos o conceito de "experiência", notaremos que o sujeito da experiência "(...) se expõe atravessando um espaço indeterminado e perigoso pondo-se nele à prova e buscando nele sua oportunidade, sua ocasião [..." (BONDÍA, 2002, p. 25). E o "perigo" nessa travessia pelo PET-Saúde pode estar exatamente no sentido da experiência e da descoberta... Experiências e descobertas que aconteceram no período crítico durante a formação. E nos aconteceram, no entanto, por estarmos inseridos em cantos inicialmente desconhecidos, com fazeres estranhos, imersos em uma realidade distante da sala de aula. Distante, mas conectada com ela... Isso, em certo sentido, referindo-nos às experiências de integração ensino-serviço, contribui para qualificar os conteúdos diversos que compõem os currículos específicos de cada núcleo da saúde, pois cria significados mais próximos aos aprendizados de cunho mais teórico.

Nesse sentido, entendemos que a prática pedagógica de integração ensino-serviço no PET-Saúde é um potente ingrediente e é estratégica para a formação de futuros profissionais que, ao menos, precisam ter noção do contexto sócio-histórico e político em saúde para melhor se situarem diante da conjetura nacional em consonância com o SUS, que é o grande provedor do campo. A produção de profissionais competentes e responsáveis é elementar também na construção participativa de uma sociedade que, de certo modo, preza o bem comum e visa contribuir para a emancipação das pessoas frente aos impasses da vida contemporânea ocidental - violência, exclusão, preconceito, inversão de valores etc.

Para os profissionais de Educação Física, para quem a presença no serviço público de saúde será cada vez maior, é necessário atenção a essas questões relativas à saúde da população, já que tradicionalmente o curso de formação não tem privilegiado muitas discussões de modo reflexivo e nem, sobretudo, o SUS (CARVALHO et al., 2013). Dessa forma, portanto, a luta também é na direção de suscitar uma preocupação maior da Educação Física em estar alinhada com os princípios norteadores da saúde no Brasil, especificamente na atenção básica, para que seja possível responder, com devida atenção e cuidado, às implicações e decorrências de dilemas nas condições de saúde e de vida da população.

\section{REFERÊNCIAS}

ABRAHÃO, Ana L. et al. A pesquisa como dispositivo para o exercício no PET-Saúde UFF/FMS Niterói. Revista Brasileira de Educação Médica, Rio de Janeiro, v. 35, n. 3, p. 435-440, 2011.

ALMEIDA, Magda M. et al. Da teoria à prática da interdisciplinaridade: a experiência do pró-saúde Unifor e seus nove cursos de graduação. Revista Brasileira de Educação Médica, Rio de Janeiro, v. 36 , n. 1, p. 119-126, 2012. Supl. 1. 
ALONSO, Andreia T.; CARVALHO, Yara M. Educação Física e PET-Saúde: relato de experiência sobre algumas das atividades na Unidade Básica de Saúde São Jorge, distrito Butantã/São Paulo/SP. In: CONGRESSO INTERNACIONAL REDE UNIDA, 10., 2012, Rio de Janeiro. Anais... Rio de Janeiro, 2012. Disponível em: < http://www.redeunida.org.br/congresso2012/anais-do-congresso >. Acesso em: 20 fev. 2015.

ANDRADE, Luiz O. M.; BUENO, Ivana C. H. C.; BEZERRA, Roberto C. Atenção primária à saúde e estratégia saúde da família. In: CAMPOS, Gastão W. S.; et al. (Org.). Tratado de saúde coletiva. 2. ed. São Paulo: Hucitec, 2009. p. 783-836.

BONDÍA, Jorge L. Notas sobre a experiência e o saber de experiência. Revista Brasileira de Educação, Rio de Janeiro, n. 19, p. 20-28, jan/abr. 2002.

BRASIL. (Constituição, 1988). Constituição da República Federativa do Brasil. Brasília: Senado Federal, 1988. Disponível em: <http://www.planalto.gov.br/ccivil 03/Constituicao/Constituicao.htm>. Acesso em: 20 fev. 2015.

BRASIL. Ministério da Saúde. Programa de Educação pelo Trabalho para a saúde (PET-Saúde), Brasília, 2008. Disponível em: <http://portalsaude.saude.gov.br/index.php/o-ministerio/principal/ secretarias/sgtes/sgtes-gestao-da-educacao/pet-saude>. Acesso em: 17 mar. 2015.

CAMPOS, Rosana O. et al. PET-Saúde como instrumento para a articulação da saúde mental e coletiva: narrativas da formação e do trabalho em saúde. Cadernos Brasileiros de Saúde Mental, Rio de Janeiro, v. 4, n. 8, p. 176-185, 2012

CARVALHO, Yara M.; CECCIM, Ricardo B. Formação e educação em saúde: aprendizados com a saúde coletiva. In: CAMPOS, Gastão W. S. et al. (Org.). Tratado de saúde coletiva. 2 ed. São Paulo: Hucitec, 2009. p. 137-170.

CARVALHO, Yara M.; PRADO, Alessandro R; ALONSO, Andreia T. Formação em educação física no Brasil: outros modos de pensar e intervir no serviço público de saúde. Educación Física y Ciencia, cidade, v. 15, n. 1, p. 10-16, 2013.

CECCIM, Ricardo B.; FEWERVWERKER, Laura C. M. O quadrilátero da formação para a área da saúde: ensino, gestão, atenção e controle social. PHYSIS: revista de saúde coletiva, Rio de Janeiro, n. 14, v. 1, p. 41-65, 2004.

CYRINO, Eliana G. et al. Ensino e Pesquisa na Estratégia de Saúde da Família: o PET-Saúde da FMB/ Unesp. Revista Brasileira de Educação Médica, Rio de Janeiro, v. 36, n. 1, p. 92-101, 2012. Supl. 1.

DIAS, Cristina M. G. C. et al. O PET-Saúde como ferramenta estratégica de implantação de um curso de medicina em uma universidade pública: relato de uma experiência. Revista Brasileira de Educação Médica, Rio de Janeiro, v. 36, n. 1, p. 76-79, 2012. Supl. 2.

FERREIRA, Vinicius S. et al. PET-Saúde: uma experiência prática de integração ensino-serviçocomunidade. Revista Brasileira de Educação Médica, Rio de Janeiro, v. 36, n.1, p. 147-151, 2012. Supl. 2.

FEUERWERKER, Laura C. M. Além do discurso da mudança na educação médica: processos e resultados. São Paulo: Editora Hucitec/Londrina: Rede Unida/Rio de Janeiro: Associação Brasileira de Educação Médica; 2002.

FONSÊCA, Graciela S.; JUNQUEIRA, Simone R. Programa de Educação pelo Trabalho para a Saúde: ressignificando a formação dos profissionais da saúde. Curitiba: Appris, 2014.

FRAGA, Alex. B.; CARVALHO, Yara M.; GOMES, Ivan M. Políticas de formação em educação física e saúde coletiva. Revista Trabalho, Educação e Saúde, Rio de Janeiro, v. 10, n. 3, p. 367-386, 2012.

MASSUDA, Adriano. Práticas de Saúde Coletiva na Atenção Primária em Saúde. In: CAMPOS, Gastão W. S.; GUERRERO, André V. P. (Org.). Manual de práticas de atenção básica: saúde ampliada e compartilhada. São Paulo: Hucitec, 2008. cap. 7, p. 179-205. 
MORAIS, Fátima R. R. et al. A importância do PET-Saúde para a formação acadêmica do enfermeiro. Revista Trabalho, Educação e Saúde, Rio de Janeiro, v. 10, n. 3, p. 541-551, nov. 2012/fev. 2013.

SANTOS, Ana L. B et al. Educação Física e o PET-Saúde: uma estratégia complementar na formação para o SUS. SANARE, Sobral, v. 10, n. 2, p. 75-78, jul./dez. 2011.

SILVA, Valdemar G. et al. A Educação Física no Programa de Educação pelo Trabalho para a Saúde de Cuiabá-MT. Revista Brasileira de Atividade Física e Saúde, Pelotas, v. 17, n. 2, p. 121-124, abr. 2012.

SOUSA NETO, Ariel et al. Programa de Educação pelo Trabalho para a Saúde nas Escolas: oficina sobre sexualidade. Revista Brasileira de Educação Médica, Rio de Janeiro, v. 36, n. 1, p. 86-91, 2012. supl. 1.

PRADO, Alessandro R.; CARVALHO, Yara M. PET Saúde - USP: As experiências dos estudantes de Educação Física. In: CONGRESSO BRASILEIRO DE SAÚDE COLETIVA, 10., Porto Alegre, 2012.

Anais... Porto Alegre, novembro, 2012. Disponível em: < https://www.abrasco.org.br/site/congressoseventos/congresso-brasileiro-de-saude-coletiva/> Acesso em: 17 mar. 2015.

TANAKA, Elisa E. et al. Projeto PET-Saúde: ferramenta de aprendizado na formação profissional em saúde. Revista Brasileira de Educação Médica, Rio de Janeiro, v. 36, n. 1, p. 136-140, 2012. supl. 2.

TEIXEIRA, Simone et al. O PET-Saúde no centro de saúde cafezal: promovendo hábitos saudáveis de vida. Revista Brasileira de Educação Médica, Rio de Janeiro, v. 36, n. 1, p. 183-186, 2012. Supl. 1.

ZEM-MASCARENHAS, Silvia H. et al. Relato da experiência de São Carlos no Programa de Educação pelo Trabalho para a Saúde. Revista Brasileira de Educação Médica, Rio de Janeiro, v. 36, n. 1, p. 142-148, 2012. Supl. 1. 